\title{
Desarrollo cognitivo y comunicación intencional preverbal: un estudio longitudinal multivariado
}

\author{
ENCARNACIÓN SARRIÁ \\ Universidad Nacional de Educación a Distancia \\ ANGel Rivière \\ Universidad Autónoma de Madrid

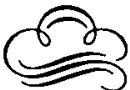 \\ Resumen
}

\begin{abstract}
A partir de la revisión de las investigaciones sobre las relaciones entre la aparición de la intención comunicativa y el desarrollo sensoriomotor, se planteó la conveniencia de realizar un estudio empírico que permitiera profundizar en el estudio de estas relaciones desde una perspectiva de análisis multivariado. Para ello se realizó un estudio longitudinal de 16 sujetos, con tres momentos de medida (en tomo a los ocho, diez y doce meses de edad). Los sujetos fueron evaluados mediante la aplicación de las escalas Uzgiris y Hunt (1975) y grabados en dos situaciones de interacción diádica, una libre con su madre y otra semiestructurada de carácter elicitador. La conducta comunicativa de los niños fue registrada mediante un sistema de codificación diseñado con tal fin (Sarriá, 1991). Los datos fueron analizados mediante análisis de correlaciones y análisis de correspondencias múltiples. Los resultados obteridos no apoyan la bipótesis de la bomología local sobre la interdependencia de determinadas capacidades cognitivas y el desamrollo comunicativo planteada por Bates, Benigni, Bretherton, Camaioni y Volterra (1979), sino más bien sugieren una bipótesis de diferenciación entre los dominios de adaptación al mundo físico y el social-comunicativo, en el periodo de aparición de la intención comunicativa.
\end{abstract}

Palabras clave: Intención comunicativa preverbal, Desarrollo social, Desarrollo sensoriomotor, Estudio longitudinal.

\section{Abstract}

From reviewing studies on the relationship between early communicative intention and sensoriomotor development in children, it appeared to be necessary to do an empirical study which would allow us to go deeper in these relationships from the view of multivariate analysis. A 16 subject longitudinal study, where measures were gotten in three different developmental moments, was carried out. Subjects were assesed by Uzgiris and Hunt's (1975) scales and they were also videotaped in two different diadic interaction situations: a free child-mother interaction, and a semi-structured eleciting one. Children's communicative bebaviour was codified by a codifying system designed on this purpouse (Samiá, 1991). Correlation and correspondence analysis were used to analyze data. Results do not support the bypothesis on the local bomology about the interdependence of some cognitive and communicative developmental abilities that Bates, Begnini, Brethertbon, Camaioni y Volterra (1979) postulated. They rather suggest a hypothesis on the differenciation between both domines: adaptation to physical world and adaptation to interpersonal-communicative world when communicative intention firstly appears.

Key words: Preverbal communicative intention, Social development, Sensoriomotor development, Longitudinal study.

Agradecimientos: Esta investigación ha sido realizada con una financiación del Centro de Investigación y Documentación Educativa.

Dirección de los autores: Facultad de Psicología. UNED. Ciudad Universitaria s/n. 28040 Madrid. 


\section{INTRODUCCION}

En la década de los setenta, una confluencia de intereses entre la psicolingǘstica y la psicología evolutiva provoca la profusión de publicaciones centradas en las primeras formas de comunicación, y más concretamente en la aparición de la comunicación intencional. Desde sus inicios, predominan en este campo las investigaciones orientadas al estudio de las relaciones entre el desarrollo comunicativo y el desarrollo cognitivo (entendido este último como adaptación al mundo físico) desde una perspectiva constructivista, de inspiración piagetiana, tanto en los constructos teóricos como en el diseño de pruebas de evaluación.

Desde el trabajo pionero de Sugarman (1973) se han sucedido diversas hipótesis explicativas, entre las que han ocupado un lugar predominante las relativas a su relación con el desarrollo de la noción de causalidad.

Sugarman (1973) parte de la hipótesis de que la interacción no verbal con las personas y la manipulación de objetos físicos son formas particulares de transacción con el entorno, sujetas ambas a un sustrato cognitivo común y, en consecuencia, con un patrón de desarrollo paralelo. En el desarrollo de estas dos facetas son identificables, a su juicio, dos momentos claves de manifestación de conductas instrumentales, la coordinación de esquemas dirigidos a personas con los dirigidos a objetos (comunicación intencional) y la coordinación objetoobjeto. Su análisis teórico le lleva a predecir qué será en el estadio V sensoriomotor (cuando el niño ya es capaz de utilizar nuevos medios y descubrirlos por experimentación activa), cuándo podrá utilizar un objeto como instrumento para conseguir otro, y cuándo su comprensión de las otras personas como agentes causales autónomos le permitirá contar con ellas, transmitiéndoles sus deseos para que le ayuden a satisfacerlos. Los resultados de su estudio empírico indican cierta coincidencia temporal entre estas dos habilidades (Sugarman, 1978, 1984).

El análisis de la comunicación intencional como una relación medios-fines en la que interviene un adulto (al que el niño debe atribuir causalidad independiente como agente) y su paralelismo con el uso de instrumentos físicos ha llevado a algunos autores a postular su relación en términos de «prerrequisitos»: el desarrollo de la noción de causalidad del estadio $\mathrm{V}$ prerrequisito necesario para la aparición de la comunicación intencional (Bates, Camaioni y Volterra, 1975; Camaioni, Volterra y Bates, 1976; Harding y Golinkoff, 1979; Harding, 1982).

En teoría, una hipótesis en términos de «prerrequisitos» recibe poco apoyo de los datos positivos (coincidencia de la presencia de la condición necesaria y del criterio) (Sugarman, 1978; Camaioni et al., 1976; Harding y Golinkoff, 1979) y es contundentemente falseada por los datos negativos (presencia de la conducta criterio con ausencia de la condición). En este sentido son especialmente relevantes los estudios de Carranza, Pérez y Brito de la Nuez (1985) y de Martisen y Von Tezchner (1988), en cuyos datos los sujetos comienzan a utilizar vocalizaciones y gestos comunicativos intencionales (dirigidos al adulto) antes de alcanzar el nivel V de la noción de intermediario (Carranza et al., 1985) o de la noción de causalidad en el uso de instrumento (Martisen y Von Tezchner, 1988).

Esta contraevidencia puede ser justificada con una diversidad de argumentos que probablemente son más complementarios que alternativos. Carranza et al. (1985) sugieren que la mayor riqueza de la experiencia del niño con las per- 
sonas podría dar lugar a que la noción de intermediario se produzca antes en relación con éstas que con los objetos físicos.

Por otra parte, una cierta reflexión sobre los estadios sensoriomotores IV y $\mathrm{V}$ puede ser útil para analizar estos resultados. Si la diferenciación entre estadios siempre tiene algo de artificial y forzado, lo es especialmente cuando se trata de delimitar fases que mantienen entre sí un alto grado de continuidad, como sucede con estos dos estadios. Los estadios IV y V suponen un importan. te avance sobre los anteriores; ambos se caracterizan por las adaptaciones sensoriomotrices inteligentes y el comportamiento intencional. En las diversas nociones de lo real (Piaget, 1936, 1937), el paso del estadio III al IV supone un cambio importante, ya que implica la transición de lo subjetivo a lo objetivo, esto es, el inicio de la objetivación del espacio, del tiempo y de la causalidad, y de la búsqueda activa en el caso de la noción de objeto. Los comportamientos propios del estadio $\mathrm{V}$ aunque también supongan un progreso decisivo, constituyen la terminación natural de los del IV. De esta forma, la base lógica que se argumenta para justificar la relación entre la comunicación intencional y el estadio $V$, sería igualmente aplicable en relación con el estadiō IV, ya que éste supone el inicio de los logros consolidados que caracterizan al estadio V. Incluso podemos pensar que el estadio IV sería un correlato más defendible si la relación se plantea con la aparición de la comunicación intencional. En este sentido nos situaríamos más cerca del criterio teórico planteado por Ainsworth y Bell (1974), Carter (1974) y Dore (1973).

Situándonos en un nivel mucho más concreto, resulta difícil el análisis crítico comparativo de las distintas investigaciones por la gran diversidad existente en aspectos tan relevantes como la definición operativa de comunicación intencional, las categorías de observación utilizadas, las tareas específicas de evaluación y los criterios de asignación a estadios o niveles.

Otra hipótesis explicativa de la contradicción entre los resultados de los diversos estudios empíricos se puede encontrar en el planteamiento que hacen Bates et al. $(1976,1977)$ en trabajos posteriores. En una reflexión más general sobre la interdependencia entre pensamiento y lenguaje estos autores definen su postura como una versión piagetiana del modelo de estructuras homólogas que implican que pensamiento y lenguaje derivan de una tercera fuente común. Esto supone que la ejecución en tareas lingüísticas y tareas cognitivas puras dependen de esquemas operativos subyacentes y compartidos por los dos dominios. Llevándolo al nivel de la adquisición de las funciones pragmáticas, el uso de instrumentos físicos y la comunicación intencional deberían analizarse como manifestaciones de la causalidad del estadio $V$, pero no existe razón alguna que permita predecir un orden concreto en la aparición de estas dos manifestaciones (Bates et al., 1976). Los objetivos de su investigación se centrarán, por tanto, en el estudio de estas estructuras compartidas. Concretamente se plantea la realización de un estudio correlacional (Bates et al., 1979) que permita la contrastación de dos diferentes hipótesis explicativas de estas relaciones: (a) un modelo de homología profunda, en el que una serie de estructuras operatorias comunes caracterizarían todas las facetas del desarrollo sensoriomotor, y (b) un modelo de homología local, que presupone la existencia de estructuras compartidas entre conductas o problemas específicos. Los resultados del estudio, en términos generales apoyan un modelo de homología local, en la medida en que las relaciones entre la frecuencia de conductas comunicativas y el nivel de desarrollo eran desiguales en las distintas nociones de desarrollo cognitivo, evalua- 
das (escalas de Üzgiris y Hunt, 1975). Concretamente, las escalas de imitación, relaciones medios-fines y juego simbólico concentraban la mayor parte de las correlaciones significativas, mientras que las de permanencia del objeto y de relaciones espaciales eran las menos relacionadas con el desarrollo comunicativo. Asimismo, el patrón temporal de las relaciones variaba entre las distintas escalas, y las correlaciones entre las diversas medidas de desarrollo cognitivo eran bajas (dato este último coincidente con los resultados presentados por Uzgiris y Hunt, 1975).

A partir de esta revisión de la bibliografía básica sobre las relaciones entre desarrollo cognitivo y la aparición de la intención comunicativa se planteó la conveniencia de realizar un estudio empírico que permitiera profundizar en el estudio de estas relaciones y su evolución durante el período de aparición de las primeras formas de comunicación intencional preverbal.

\section{ESTUDIO EMPIRICO}

2.1. Objetivos. El estudio se planteó con el objetivo básico de analizar las relaciones entre el uso de las primeras formas de comunicación intencional (y sus características) y otras facetas del desarrollo sensoriomotor. Concretamente se pretendía la contrastación de la hipótesis de homología local propuesta por Bates et al. (1979) desde una perspectiva de análisis multivariado, que consideramos más adecuada que la perspectiva correlacional bivarada utilizada por estos autores. Asimismo, entre los objetivos del estudio se incluía la creación de un sistema de categorías que permitiera la observación y registro de la comunicación intencional preverbal con garantías de rigor, pero sin pérdida del significado esencial de dicha conducta.

2.2. Diseño. El estudio realizado responde a las características del diseño longitudinal de medidas repetidas con intervalos de medidas iguales (dos meses aproximadamente). En concreto, siendo la edad una de las variables de interés se siguió el desarrollo de una muestra de 16 sujetos, obteniendo tres puntos de media para cada sujeto: en torno a los ocho, los diez y los doce meses de edad. Los datos específicos de los intervalos y la media correspondiente a cada uno de los momentos de medida se encuentran en la Tabla I.

2.3. Sujetos. Se partió de una muestra inicial de 22 sujetos, pero diversas circunstancias provocaron una mortandad experimental de seis de ellos, quedándonos con una muestra definitiva de 16 niños, ocho varones y ocho niñas. Las condiciones previas para la selección de los sujetos fueron: parto normal, ausencia de problemas físicos en el niño y de sociales en su entorno. Desde un

TABLA I

Edad media e intervalos de muestra en los tres momentos de estudio.

\begin{tabular}{|c|c|c|}
\hline Medida & Intervalo (meses/días) & $\mathbf{x}$ \\
\hline $1{ }^{a}$ medida & $7,27-8,19$ & 8,8 \\
$2 .^{a}$ medida & $9,28-10,18$ & 10,7 \\
$3{ }^{a}$ medida & $11,15-12,13$ & 12,2 \\
\hline
\end{tabular}


principio se buscó la hetereogeneidad en la composición de la muestra, en pro de su mayor representatividad. La muestra definitiva presenta las siguientes características:

1. Sexo: $50 \%$ varones y $50 \%$ niñas.

2. Orden de nacimiento: $62,5 \%$ primogénitos y $37,5 \%$ no primogénitos.

3. Nivel socioeconómico familiar (evaluado por el trabajo del padre): $68,75 \%$ clase media, $25 \%$ obrero especializado y $6,25 \%$ peonaje.

4. Situación laboral de la madre en el primer año de vida del niño: el $43,75 \%$ trabajan fuera del domicilio y el $56,25 \%$, no.

2.4. Procedimiento. Las sesiones de recogidas de datos se realizaron en los domicilios de los sujetos, dada su corta edad y para facilitar su espontaneidad.

En cada uno de los momentos de medida, los sujetos fueron evaluados mediante las escalas Uzgiris y Hunt (1975), asignándoseles una puntuación en cada una de las siete subescalas en función del rendimiento máximo alcanzado en las tareas de prueba.

Además de la evaluación, cada sujeto fue grabado en vídeo en dos situaciones de interacción social (ambas de 20 minutos de duración): una situación de interacción libre con su madre (situación libre), y otra situación de interacción social semiestructurada con una investigadora (situación elicitadora). En este caso la investigadora actuaba según un guión flexible predeterminado en el que se había incluido una selección de juegos y materiales potencialmente elicitadores de conductas comunicativas en el niño (ver Sarriá, 1989).

Las grabaciones de vídeo fueron utilizadas para la observación y registro de las conductas comunicativas. Este se efectuó con la aplicación de un sistema de codificación para la observación de la comunicación intencional preverbal (Sarriá, 1989, 1991), diseñado con tal fin y que permitía el registro de los actos comunicativos intencionales, con identificación específica de su función, las conductas concretas utilizadas y sus características espacio-temporales y sociales. La unidad de análisis fundamental era el «acto comunicativo intencional», definido, como una categoría de límites borrosos, por medio de los atributos característicos de sus prototipos.

Definimos el Acto comunicativo intencional preverbal (ACI) como un grupo de conductas que en conjunto poseen la capacidad efectiva de transmitir un mensaje (a un receptor destinatario del mismo), y que son realizadas por un sujeto (emisor), de forma voluntaria con ese fin, bien siendo éste el único objetivo o integrado junto con otros objetivos.

El contenido de esta definición se manifiesta en una serie de propiedades del acto comunicativo intencional, fácilmente perceptibles. Suponiendo que la categoría de ACI constituye una categoría natural, los atributos que ahora señalaremos no van siempre todos unidos, ni son universales para todos los elementos pertenecientes a esa categoría, pero están los suficientemente generalizados como para ser útiles en la identificación de actos comunicativos intencionales.

A. Estructuración interna: El ACI está constituido por un conjunto de conductas que se presentan con cierta organización. Entre estas conductas podemos distinguir un elemento máximo, que es el elemento más constante, constituyendo el núcleo del acto, y diversos elementos variables, de menor duración en sus apariciones, y que se vinculan con el elemento máximo.

Esta estructuración interna nos permite delimitar y diferenciar los ACIs, en función del cambio de la conducta que se constituye en elemento máximo. 
B. Elicitador: Este conjunto de conductas elicitan cambios de conducta en la persona que los presencia. Se produce una contiguiidad temporal entre las acciones de los dos sujetos, estando éstas relacionadas entre sí por algún elemento común (objeto, acción...). Esta manifestación sólo es esperable en condiciones de atención del otro sujeto distinto al emisor del ACI.

C. Persistencia de la conducta del emisor del ACI: El niño persiste en su conducta, añadiendo, intesificando o sustituyendo señales, de forma contingente a la conducta del receptor, hasta que el objetivo se consigue o el fracaso es evidente. La persistencia tiene al menos dos manifestaciones distintas y complementarias:

a) Nivel micro: se manifiesta en la duración del elemento máximo del ACI, siendo ésta igual o superior a dos segundos.

b) Nivel macro: se manifiesta en la invariancia del objeto referente en el transcurso de una sucesión de ACIs, provocada por la ineficacia del anterior.

Si estos ACIs sucesivos son iguales, es decir, no hay cambio en el elemento máximo, sino que los cambios se refieren a la intensificación de las acciones y/o la alteración de su orden, hablamos de persistencia inflexible. Si estos ACIs sucesivos son distintos (cambio en elemento máximo), hablamos de persistencia flexible.

D. Existencia de un referente (objeto físico o acción) objetivable y claramente determinado por las conductas constituyentes del ACI emitido: Las acciones relevantes constituyentes del ACI están dirigidas específicamente al objeto referente y al sujeto receptor del ACI, bien de forma simultánea o sucesiva; o todas ellas dirigidas al receptor. Los ACIs no se componen de conductas dirigidas simultáneamente a distintos puntos u objetos. Por ejemplo, no parece considerable como ACI un acto en el cual el niño entrega un objeto al adulto, y no mira ni al objeto ni al adulto, sino que mira a otro objeto distinto; tampoco es un ACI el acto en el que el niño mira un objeto y su dedo índice estirado está señalando otro punto desatendido (por ejemplo a su propio zapato).

E. Alguna de las acciones relevantes, constituyentes del ACI, está dirigida al sujeto destinatario del mismo: En la mayoría de los casos la conducta dirigida al otro, y la más clarificadora, es la mirada a los ojos, pero no es la única posible. Por ejemplo, cuando el emisor entrega un objeto al adulto, no siempre mira a los ojos sino que puede permanecer atento a las manos del mismo si espera que haga algo con el objeto, y sólo si el adulto no reacciona utilizará la mirada a los ojos para clarificar aún más su intención. Otro ejemplo puede ser cuando el niño acerca un objeto al lugar apropiado de la cara para solicitar una acción con él (vgr. acerca una flor a la nariz para que la huela), en este caso puede que no haya una mirada concreta a los ojos, sino que permanezca atento a la cara de forma general.

F. Carencia de funcionalidad directa (sobre un posible objeto físico) de las acciones relevantes de $A C I$ : Las acciones relevantes del ACI implican una serie de movimientos que serían insuficientes para cumplir su objetivo si actuaran directamente sobre el objeto físico; bien por su carácter fragmentario (por ejemplo, al estirar el brazo hacia un objeto deseado, como parte de un ACI, se hace de forma insuficiente para alcanzarlo), o bien por su carácter gestual o expresivo (por ejemplo, indicación con el índice, sonrisa, contacto ocular...). 
El registro fue contínuo y se procedió al recuento de frecuencias de aparición de las distintas categorías de conductas relevantes (ver listado de símbolos en el anexo).

La fiabilidad interobservadores, se controló inicialmente por «consenso» entre los dos observadores. De las sesiones restantes se eligieron aleatoriamente veinte, que fueron codificadas de forma independiente por los dos observadores y cuyos registros fueron contrastados para calcular el grado de acuerdo existente. Para ello se utilizó el índice $\pi$ (pi), (Scott, 1955), pero con las mejoras en el cálculo de la proporción de acuerdo esperado propuestas por Blanco y Anguera (1984).

Dado que la tarea de codificación se realizó registrando también el orden de aparición de los diferentes actos comunicativos intencionales, el cálculo del índice $\pi$ (pi) se pudo aplicar teniendo en cuenta el orden como criterio de acuerdo. Las omisiones se valoraron como errores de desacuerdo. Los criterios utilizados son de un carácter más conservador del estrictamente necesario (Bakeman y Gottman, 1986) ya que los datos fueron posteriormente tratados e interpretados básicamente en términos de frecuencia de aparición de las categorías, pero dada la novedad del sistema de codificación se consideró conveniente.

El carácter de reclasificación del sistema de codificación y la decisión de tener en cuenta el orden del registro en el cálculo del acuerdo entre los observadores, determina la existencia de un índice $\pi$ (pi) por sesión y dimensión de clasificación. En lo que se refiere al registro de ACIs, su función y las conductas comunicativas implicadas en el acto, los índices obtenidos varían entre 0.65 y 1 , que se pueden considerar satisfactorios.

2.5. Análisis e interpretación de los datos. Para la comprobación de las hipótesis relativas a la relación entre el desarrollo cognitivo y el comunicativo, se procedió a la aplicación de análisis bivariados y multivariados, dentro de las posibilidades que permitían los datos. En concreto, análisis de correlación de Spearman, análisis de correspondencias múltiples. Los datos procedentes de las dos situaciones de interacción registradas se analizaron siempre por separado. Se trataron las variables relativas a las diferentes escalas Uzgiris y Hunt (1975) (consideradas medidas del desarrollo cognitivo de los sujetos) y las medidas de frecuencia de aparición de las categorías de ACIs, Rs, funciones y conductas comunicativas; para ello, los datos de observación se transformaron previamente.

Se planteó la posibilidad de la transformación de los datos dada la diversidad de tipo y la diferencia de amplitud de las escalas, de las variables a relacionar: en el caso de las variables cognitivas, disponíamos de una escala de tipo ordinal con una amplitud de una a diez; en el de las variables comunicativas, una escala de intervalo con amplitud de cero a cuarenta. Para ello, las frecuencias de aparición de las categorías de comunicación se agruparon en intervalos de amplitud 5, y a cada uno de los intervalos se le asignó un valor comprendido entre 2 y 10 en orden creciente (el 1 se reservó para reflejar el valor 0 , que planteaba problemas en ciertos programas de análisis de datos). De esta forma, estas variables, que anteriormente correspondían a escala, de intervalo, se convierten en variables ordinales, y disminuye considerablemente la amplitud de la escala con lo que se aproxima y asemeja a la escala de las variables cognitivas.

2.5.1. Análisis de correlaciones. Se planteó la conveniencia de realizar análisis de correlaciones entre las puntuaciones obtenidas en las diversas escalas de desarrollo cognitivo y las frecuencias de conductas comunicativas para facili- 
tar la comparación inicial con los resultados obtenidos por Bates et al., (1979). Se realizaron mediante el cálculo de índices de correlación de Spearman, (BMDP3S) dado el carácter ordinal de las variables de evaluación. Las correlaciones estadísticamente significativas con $\mathrm{p}(\alpha) \leqslant 0.05$ se reflejan en las tablas resumen II, III y IV.

Un primer aspecto a destacar es la baja proporción de correlaciones estadísticamente significativas. Centrándonos, por ejemplo, en las relaciones entre las escalas de tareas cognitivas y los ACIs, las respuestas y las funciones comunicativas, vemos que de las trescientas setenta y ocho correlaciones calculadas (nueve categorías por siete escalas, por tres momentos, por dos situaciones) sólo doce alcanzan el nivel de significación; esto es, un 3,17\%. Resalta además la especial carencia de correlaciones significativas en los datos de los doce meses, que arrojan una sola correlación significativa (nivel de esquemas sensoriomotores con frecuencia de protestas), y la ausencia total de correlaciones significativas con la escala II (Relaciones medios-fines).

En lo que se refiere a las relaciones de estas escalas con las conductas comunicativas utilizadas en los ACIs, la proporción de correlaciones estadísticamente significativas continúa siendo muy escasa, el $3.46 \%$. En este caso, de las seiscientas noventa y tres correlaciones halladas sólo alcanzan el nivel de significación estadística, venticuatro; y de éstas, diez son negativas. Es de destacar que la escala II (Relaciones medios-fines) tampoco presenta relaciones suficientemente potentes con las conductas comunicativas. La escala con mayor número de correlaciones en las tablas es la escala VI de esquemas sensoriomotores, aunque casi la mitad son negativas.

La realización de estos análisis de correlación tenía como objetivo fundamental la contrastación de los resultados obtenidos por Bates et al. (1979), y por tanto, el sometimiento a prueba de la hipótesis de homología local vinculada a ellos. Esta contrastación cobraba un sentido especial dado que en su estu-

\section{TABLA II}

Correlaciones de Spearman estadísticamente significativas con $p(\alpha) \leq 0.05$, entre las escalas de tareas cognitivas y categorias de observación sobre funciones y conductas comunicativas, a los ocho meses.

\begin{tabular}{|l|c|c|c|c|}
\cline { 2 - 5 } \multicolumn{1}{c|}{} & \multicolumn{2}{c|}{ Situación elicitadora } & \multicolumn{2}{c|}{$\begin{array}{c}\text { Situación de interacción } \\
\text { libre }\end{array}$} \\
\cline { 2 - 5 } \multicolumn{1}{c|}{} & $\begin{array}{c}\text { Funciones } \\
\text { comunicativas }\end{array}$ & $\begin{array}{c}\text { Conductas } \\
\text { comunicativas }\end{array}$ & $\begin{array}{c}\text { Funciones } \\
\text { comunicativas }\end{array}$ & $\begin{array}{c}\text { Conductas } \\
\text { comunicativas }\end{array}$ \\
\hline I & & & ACI (.610) & \\
\hline II & & & & CO (-.534) \\
\hline IIIA & & & & VO (-.585) \\
\hline IIIB & & MA (-.597) & DO (.611) & \\
\hline IV & & & ACI (.688) & \\
\hline V & NO (.571) & OF (-.666) & DO (.571) & \\
\hline VI & & TO (.629) & & CO (.866) \\
\hline
\end{tabular}


dio correlacional Bates et al. (1979) abandonan los protoimperativos y protodeclarativos (Bates et al., 1975) como unidades de análisis, registrando una serie de conductas y funciones comunicativas en las que sólo se emplea su diferenciación en ritualizadas y no ritualizadas para valorar la intencionalidad del comportamiento comunicativo del niño. Sin embargo, en la investigación que nos ocupa, el registro de funciones y conductas comunicativas se realiza con

\section{TABLA III}

Correlaciones de Spearman estadísticamente significativas con $p(\alpha) \leq 0.05$, entre las escalas de tareas cognitivas y categorias de observación sobre funciones $y$ conductas comunicativas, a los diez meses.

\begin{tabular}{|c|c|c|c|c|}
\hline & \multicolumn{2}{|c|}{ Situación elicitadora } & \multicolumn{2}{|c|}{$\begin{array}{c}\text { Situación de interacción } \\
\text { libre }\end{array}$} \\
\hline & $\begin{array}{c}\text { Funciones } \\
\text { comunicativas }\end{array}$ & $\begin{array}{c}\text { Conductas } \\
\text { comunicativas }\end{array}$ & $\begin{array}{c}\text { Funciones } \\
\text { comunicativas }\end{array}$ & $\begin{array}{c}\text { Conductas } \\
\text { comunicativas }\end{array}$ \\
\hline I & $\mathrm{R}(-.598)$ & & & $\mathrm{GC}(-.576)$ \\
\hline \multicolumn{5}{|l|}{ II } \\
\hline IIIA & $\mathrm{DO}(.553)$ & & & \\
\hline IIIB & & & & OF $(-.564)$ \\
\hline IV & DO (.516) & $\begin{array}{l}\text { MA }(.540) \\
\text { ES }(.516)\end{array}$ & NO (.572) & $\mathrm{TA}(.511)$ \\
\hline $\mathrm{V}$ & $\mathrm{DO}(.560)$ & $\begin{array}{l}\text { SE (.683) } \\
\text { EV }(.683)\end{array}$ & & AP (.683) \\
\hline VI & & $\mathrm{CO}(-.516)$ & $\mathrm{DO}(-.633)$ & $\begin{array}{c}\text { MA }(-.622) \\
\text { ES }(-.626)\end{array}$ \\
\hline
\end{tabular}

\section{TABLA IV}

Correlaciones de Spearman estadisticamente significativas con $p(\alpha) \leq 0.05$, entre las escalas de tareas cognitivas y categorias de observación sobre funciones y conductas comunicativas, a los doce meses.

\begin{tabular}{|c|c|c|c|c|}
\hline & \multicolumn{2}{|c|}{ Situación elicitadora } & \multicolumn{2}{|c|}{$\begin{array}{c}\text { Situación de interacción } \\
\text { libre }\end{array}$} \\
\hline & $\begin{array}{c}\text { Funciones } \\
\text { comunicativas }\end{array}$ & $\begin{array}{c}\text { Conductas } \\
\text { comunicativas }\end{array}$ & $\begin{array}{c}\text { Funciones } \\
\text { comunicativas }\end{array}$ & $\begin{array}{c}\text { Conductas } \\
\text { comunicativas }\end{array}$ \\
\hline \multicolumn{5}{|l|}{ I } \\
\hline \multicolumn{5}{|l|}{ II } \\
\hline IIIA & & $\begin{array}{c}\mathrm{V}(.647) \\
\operatorname{EV}(.671)\end{array}$ & & EN (.524) \\
\hline IIIB & & TO (.522) & & MA (-.638) \\
\hline \multicolumn{5}{|l|}{ IV } \\
\hline \multicolumn{5}{|l|}{$\mathrm{V}$} \\
\hline VI & & ES (.566) & NO $(.516)$ & V (.516) \\
\hline
\end{tabular}


la previa identificación de su carácter de acto comunicativo intencional o su implicación en él. Interesaba, por tanto, comprobar si el patrón de relaciones específicas obtenido en las matrices de correlaciones de Bates et al. (1979) se mantenía al introducir este filtro de intencionalidad comunicativa de la conducta.

Revisando las correlaciones estadísticamente significativas obtenidas en nuestro estudio, tenemos que afirmar que, en esencia, el patrón de relaciones específicas observado por Bates et al. (1979) no se reproduce en nuestros resultados. Destaca en estas diferencias la ausencia total de correlaciones significativas de las medidas comunicativas con la escala de Relaciones medios-fines.

Nuestros resultados coinciden con los de Bates et al. (1979) en las variaciones de las correlaciones estadísticamente significativas en los distintos momentos de estudio. Pero la escasez de correlaciones significativas en todos ellos (el porcentaje no llega al $4 \%$ ) y su dispersión entre las distintas escalas (al margen de la ausencia absoluta de correlaciones con la escala II, ya comentada) no nos permite hablar de relaciones específicas que contribuyan a sostener la hipótesis de la homología local sobre la interdependencia estructural entre las capacidades comunicativas y ciertas capacidades sensoriomotrices cognitivas.

$\mathrm{La}$ escasa coincidencia de correlaciones significativas entre los datos de la situación elicitadora y los de la situación de interacción libre de nuestro estudio es, ya por sí misma, bastante desconcertante. No resultan especialmente extrañas entonces las diferencias existentes cuando se compara con los datos de otra investigación. Quizás más que hablar de diferencias en los resultados debería tratarse en términos de inconsistencia de los mismos.

Hacia esta idea apuntan también las conclusiones de Hogan (1983) y Bates y Snyder (1987) en sus revisiones teóricas de las investigaciones sobre la relación entre capacidades cognitivas y desarrollo comunicativo. Entre sus observaciones destaca el hecho de que cuando la revisión de estudios incluye también aquellas investigaciones sobre las primeras formas de comunicación verbal, cada una de las escalas de Uzgiris y Hunt (1975) se ha mostrado, al menos en una ocasión, como la más claramente relacionada con el desarrollo comunicativo (Hogan, 1983).

Por otra parte, como vemos en las tablas, aunque dominan las correlaciones positivas, también aparecen algunas negativas estadísticamente significativas. Este dato que en principio puede resultar desconcertante tiene, a nuestro juicio, fácil explicación. Dado el gran número de correlaciones calculadas, no es extraño que algunas de ellas alcancen valores estadísticamente significativos aunque sea solamente por azar (Martín y Bateson, 1986), y esto puede ser así tanto para las correlaciones positivas como para las de signo negativo. Lo que nos lleva a poner en cuestión el valor real de significación teórica de las correlaciones estadísticamente significativas obtenidas en este estudio y en otros cuyas matrices de correlaciones son igualmente desmesuradas para los resultados obtenidos (vgr., el estudio de Bates et al., 1979).

Bates et al. (1979) justifican la aplicación del análisis de correlaciones, para la comprobación de esta hipótesis, con el supuesto de que si dos dominios son estructuralmente interdependientes, entonces su desarrollo paralelo se manifestaría en la concordancia de los niveles mostrados por los sujetos en dichos dominios. Sin embargo, las propias limitaciones del análisis de correlaciones puede estar dificultando la contrastación de estas hipótesis. Podría ser conveniente proceder a la aplicación de análisis multivariados para profundizar en el estudio de este problema. 
2.5.2. Análisis de correspondencias múltiples. Con el fin de explorar las relaciones entre las funciones y las conductas comunicativas observadas con el nivel de desarrollo demostrado en las escalas sobre tareas cognitivas, se aplicaron análisis de correspondencias múltiples, analizando cada momento evolutivo estudiado y cada situación por separado, haciendo uso del S.P.A.D. (Lebart y Morineau, 1984) en sus etapas DONNE, LILEX, MULDI y GRAPH.

Como variables comunicativas se incluyeron las diferentes funciones, las respuestas y las conductas comunicativas. No se incluyó el total de ACIs por su carácter de sumatorio de las distintas funciones. También quedaron excluidos de estos análisis las categorías sobre las características de los ACI y respuestas, en pro de una mayor claridad de los factores. También en esta ocasión se utilizaron los datos de frecuencia de aparición transformados.

Por razones de espacio evidentes, debemos renunciar a reflejar directamente la información sobre las coordenadas, contribuciones y valores de test de cada variable y modalidad respecto a cada factor, así como las representaciones gráficas de los cruces de factores resultantes y que fue utilizada para interpretar la estructura factorial de los datos. Presentaremos, por tanto, un resumen de los resultados de los seis análisis de correspondencias aplicados, sin especificar la composición concreta de cada uno de los factores (para más detalle ver Sarriá, 1989).

En los resultados de la aplicación del análisis de correspondencias múltiples a los datos de la situación elicitadora, observamos que sistemáticamente, en cada uno de los momentos estudiados, un tercio de los seis factores más explicativos tienen una composición (según las modalidades de contribución positiva) exclusiva de uno de los dominios: cognitivo o comunicativo (del conjunto de los factores de un solo dominio, el $50 \%$ son del dominio cognitivo y el otro $50 \%$ del comunicativo).

Asimismo, y en los tres momentos analizados, la escala de «tareas cognitivas» que más frecuentemente aparece (con contribución positiva), formando parte de los factores, es la escala de imitación verbal, aunque destaca también la de imitación gestual. Curiosamente, éstas son algunas de las escalas más sociales (junto con la IV) y menos definibles como «tareas cognitivas con objeto», de las siete estudiadas.

En cuanto a las funciones comunicativas, en los datos de los ocho y los diez meses son especialmente característicos los ACIs reguladores de interacción con o sin objeto implicado, mientras que los doce meses aparecen especialmente caracterizados por las demandas de ayuda y los protodeclarativos, manteniéndose la presencia de ACIs de juego interactivo con objeto y desapareciendo los ACIs reguladores de interacción sin objeto.

Por otra parte, el factor 1 de los datos de los ocho meses llama la atención por la existencia de gran número de modalidades de tareas cognitivas (aparecen seis de las siete escalas) en su composición, mientras que el factor 1 de los doce meses es mayoritariamente comunicativo (doce variables comunicativas), lo que podría ser interpretado como un indicador del progreso experimentado en esta faceta del desarrollo, de ahí su mayor peso en la explicación de la varianza de los sujetos.

Si analizamos los resultados generales del análisis de correspondencias múltiples aplicado a los datos de la situación de interacción libre, observamos que, en cuanto a las variables comunicativas, también en estos datos se manifiesta una gran diferencia en el comportamiento de los sujetos a los doce meses frente 
a los otros dos momentos estudiados. Esto se refleja en la composición de los factores, ya que, tanto a los ocho como a los diez meses, las variables comunicativas aparecen casi exclusivamente en su modalidad de ausencia; sin embargo, a los doce meses contribuyen positivamente modalidades de uso frecuente de ACIs de demanda de objeto, protodeclarativos, solicitud de juego interactivo con objeto, demanda de ayuda y protestas.

Respecto a las puntuaciones de las escalas de tareas cognitivas que componen los factores, no existen constancias ni cambios relevantes dignos de comentar, más allá de la esperada relación edad-puntuación; esto es, las puntuaciones de las escalas que aparecen en los factores de los ocho meses son relativamente más bajas que las presentes en los diez y doce meses, disminuyendo esta diferencia al comparar estos dos últimos momentos estudiados.

En resumen, en los resultados de la aplicación de análisis de correspondencias múltiples se manifiesta, tanto en los datos de la situación libre como en los procedentes de la situación elicitadora, un aumento en la capacidad explicativa de la varianza por parte de las variables comunicativas. Este aumento aparece claramente al comparar los resultados de los ocho meses con los relativos a los doce meses, aunque se refleja de forma diferente en los datos de una y otra situación. Así, por ejemplo, con los datos de la situación elicitadora el factor 1 de los ocho meses es de composición fundamentalmente cognitiva, y sin embargo, a los doce meses, el factor 1 es fundamentalmente comunicativo. Por su parte, los datos de la situación libre a los ocho meses presentan cuatro de los seis factores más potentes con una composición esencialmente cognitiva, ya que las modalidades de las variables comunicativas que aparecen (cuando aparecen) reflejan la ausencia de dichas conductas; mientras que a los doce meses, los factores de un solo dominio en su composición se refieren al dominio comunicativo.

Otro elemento común en los resultados procedentes de estas dos situaciones de observación es la importante proporción de factores de composición exclusiva o fundamental de un solo dominio, ya sea cognitivo o comunicativo. En los datos de la situación elicitadora, los factores de un solo dominio suponen un tercio de los seis principales factores, y esto se mantiene constante en los tres momentos estudiados. En los datos de la situación libre, el porcentaje de factores de un solo dominio varía desde el $50 \%$ en los datos de los doce meses hasta el $100 \%$ en los datos de los diez. El peso explicativo de los factores de un solo dominio y su especial predominio en el caso de los datos de la situación de interacción libre se refleja en la Figura 1.

En este histograma de barras se representa esta información organizada teniendo en cuenta el porcentaje de varianza explicada por cada factor y diferenciando los tres tipos de factores considerados: cognitivo (composición exclusiva de este dominio), comunicativo (composición exclusiva del dominio comunicativo) y mixto (definido por modalidades de ambos dominios).

Ahora bien, antes de dar por terminada la descripción de los resultados de estos análisis y proceder a una interpretación más general, es preciso recordar que la mayoría de los factores tienen bajo poder explicativo de la varianza.

Recordemos que la aplicación del análisis de correspondencias múltiples para el tratamiento de los datos de este estudio se justifica, entre otras razones, por el interés en las hipótesis de homología local vs. homología profunda planteadas por Bates et al. (1979), ya que el análisis de correspondencias a través 
de la organización de las variables en factores puede reflejar el tipo de relaciones existentes entre ellas.

Podemos decir que el modelo de homología profunda supone la existencia de una serie de estructuras organizativas comunes a todos los dominios y la noción de estadios únicos con los consiguientes puntos de transición generales en el desarrollo de las diversas nociones. Esta concepción llevaría a predecir la aparición de factores que estarían formados por un conjunto muy amplio de variables representantes de los diversos dominios, y que se reproducirían con cierta constancia en los diversos momentos de estudio (ocho, diez y doce meses).

Por su parte, el modelo de homología local postula la existencia de estructuras subyacentes específicas de dominios particulares y de estadios sucesivos que van siendo alcanzados dentro de los dominios. Este modelo predeciría, por tanto, la aparición de diversos factores, compuestos cada uno de ellos por variables concretas que reflejan su estructura subyacente común, y con variaciones según el momento de estudio de los sujetos.

En general, teniendo en cuenta el conjunto de los resultados obtenidos en los diversos análisis de correspondencias múltiples, no se puede afirmar que estos resultados apoyen una hipótesis de homología profunda. La peculiar y cambiante composición de los factores se acerca más al planteamiento de homología local; pero, al mismo tiempo, la abundancia de factores compuestos exclusivamente por variables cognitivas y otros por variables comunicativas lleva a poner en duda la existencia de estructuras compartidas entre las capacidades vinculadas al dominio del mundo físico y las capacidades de adaptación al mundo social. Ahora bien, esto no niega la posibilidad de la existencia de homologías

\section{FIGURA I}

Representación gráfica del \% de varianza explicada por los 6 primeros factores resultantes de la aplicación de análisis de correspondencias múltiples en los tres momentos estudiados.

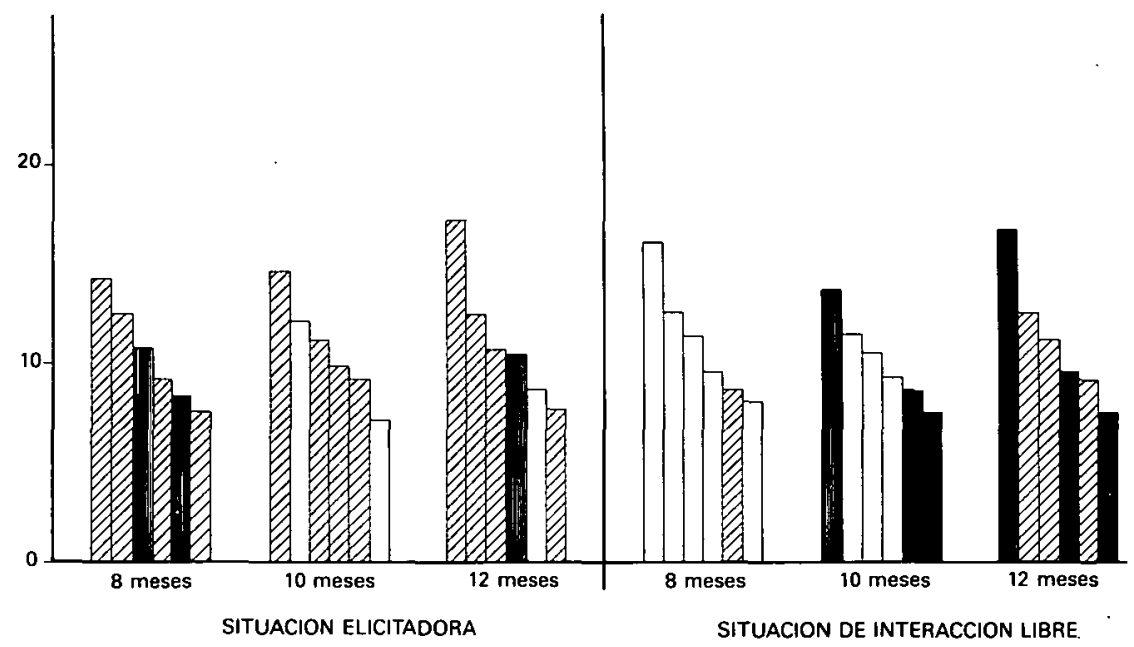


locales entre las diversas capacidades cognitivas, tal y como refieren Uzgiris y Hunt (1975) y Bates et al. (1979), y confirman nuestros propios análisis de correlación entre las siete subescalas de Uzgiris y Hunt (1975). La escasez de correlaciones estadísticamente significativas entre las diversas subescalas (Sarriá, 1989) coincide con los resultados de estas dos investigaciones a pesar de las variaciones existentes en los criterios de evaluación (en nuestro caso hemos utilizado los propuestos por Uzgiris y Hunt, 1975) y que sin embargo son alterados en la investigación de Bates et al. (1979).

Respecto a la organización en factores con composición de un solo dominio cognitivo o comunicativo, no podemos comparar los datos con los de las autoras de la hipótesis que pretendíamos comprobar, ya que no llegan a realizar este tipo de análisis ni similares. Hemos localizado, sin embargo, una investigación de referencia que, de alguna forma, respalda nuestros resultados: esta se basa en la aplicación de análisis factorial confirmatorio para el estudio de las relaciones entre las capacidades sensoriomotoras y comunicativas en la primera infancia, realizada por Hogan (1983).

Hogan (1983) planteó un estudio con ciento cincuenta niños normales divididos en dos grupos: (1) sesenta y un niños con edades mentales entre ocho y trece meses, y (2) sesenta y nueve niños entre catorce y ventiún meses de edad mental. Evaluó a los sujetos mediante la aplicación de las esclas ESCS de Seibert y Hogan (1982) y una adaptación de las escalas Uzgiris y Hunt, AUHS (Mundy y Seibert, 1980). La adaptación consistía en la eliminación de las escalas de Imitación y de los ítems sociales de la escala de Causalidad operativa. Los criterios de evaluación de todas las escalas se concretaban en la asignación de los sujetos a determinados niveles o estadios acordes con el modelo teórico subyacente a las escalas.

Mediante el análisis factorial confirmatorio de modelos estructurales (programa LISREL-V), se sometió a prueba el ajuste de diferentes modelos teóricos a los datos obtenidos. Los modelos teóricos variaban en el número de factores y su contenido. Los resultados indicaron que un modelo de un único factor general que incluyera todas las escalas (tanto las sociales-comunicativas como las cognitivas) no se ajustaba a los datos de ninguno de los grupos. Concretamente el mejor ajuste a los datos del grupo de niños con edades mentales entre ocho y trece meses (que coincide con el período de estudio de nuestro trabajo), era un modelo factorial múltiple de tres factores latentes: Cognitivo, Social y Comunicativo. Los indicadores del factor cognitivo eran las cinco escalas adaptadas de Uzgiris y Hunt; los del factor social, las tres escalas de la dimensión funcional de interacción social de las ESCS, es decir, MSI, RSI e ISI, y los indicadores del factor comunicativo, las restantes escalas ESCS, relativas a las dimensiones de atención compartida y regulación conductual. El mejor ajuste obtenido por este modelo demuestra, a juicio de Hogan (1983), la diferenciación de estos tres dominios en el primer año de vida del niño.

Salvando las distancias de las formas de medida de uno y otro estudio, estos resultados son consistentes con la numerosa presencia de factores de composición de un solo dominio (cognitivo o comunicativo) en los resultados de los análisis de correspondencias múltiples.

Si nos distanciamos algo más en el tipo de estudio e incluso en el momento evolutivo considerado, se pueden encontrar diversas investigaciones que plantean la diferenciación de la adaptación al mundo físico de los objetos y la adaptación al mundo social. El desfase temporal en los niveles de permanencia de 
objeto cuando ésta se refiere a objetos físicos o a personas (Bell, 1970; Jackson et al., 1978; Pardise y Curcio, 1974; Saint-Pierre, 1962; Seibert, 1976), la respuesta diferencial en la percepción de contingencias cuando éstas implican un estímulo social o uno no social (González del Yerro, 1986) y los datos de investigación con primates no humanos (Gómez, 1990) son algunas de las diversas señales encontradas de esta diferenciación.

En este sentido puede ser ilustrativo tener en cuenta la investigación sobre autismo.

Una de las investigaciones con resultados más claros sobre la relación específica entre la comunicación intencional y la comprensión de la causalidad física en el uso de objetos es la realizada por Curcio (1978) con una muestra de sujetos autistas. Aunque con algún dato negativo, casi la totalidad (cinco de seis) de los niños que se comunicaban intencionalmente (con un patrón de conducta comunicativa que incluía contacto ocular con el adulto) mostraban el nivel de estadio $\mathrm{V}$ en la comprensión de la causalidad (evaluados mediante las escalas II, IV de Uzgiris y Hunt, 1975), mientras que los niños que utilizaban estrategias de solicitud de ayuda más primitivas (carentes de contacto ocular con el otro), se situaban en un nivel inferior al estadio $\mathrm{V}$ en alguna de estas dos escalas. Pero los resultados de esta investigación no suponen necesariamente una nueva contradicción en la evidencia empírica acumulada. La peculiaridad de la muestra utilizada puede ser justificación suficiente para esta discrepancia de los datos. Estos resultados pueden llegar a convertirse incluso en un apoyo indirecto de la hipótesis de la diferenciación de dominios al considerar los resultados de algunas investigaciones comparativas entre autistas y normales.

Un estudio realizado por Baron-Cohen, Lesie y Frith (1986) muestra que cuando a sujetos normales se les plantea una tarea que consiste en ordenar viñetas, siguiendo un criterio de relaciones de causalidad psicológica, o un criterio de causalidad física, ordenan mejor la secuencia de causalidad psicológica. Sin embargo, el grupo de sujetos autistas, ante la misma tarea, ordenan mejor las secuencias de causalidad física.

Otro estudio comparativo oportuno para esta reflexión, ha sido realizado recientemente por Rivière y Castellanos (en prensa), y compara el rendimiento de sujetos autistas y normales en tareas de conocimiento personal y compresión interpersonal. En este estudio un grupo de niños normales de cinco años y un grupo de autistas inteligentes fueron evaluados con tareas de habilidades de tipo operatorio y con tareas que implican comprender cómo actuaría alguien en función de un estado de conocimiento. Los resultados indican que en los niños normales no hay relación entre un tipo de habilidades y otro, mientra que en los sujetos autistas hay una dependencia estricta entre su capacidad de resolver la tarea interpersonal y la capacidad para resolver la tarea operatoria. Entre los sujetos autistas sólo resuelven la tarea interpersonal aquellos que también resuelven la operatoria; sin embargo, los niños normales resuelven correctamente la tarea interpersonal sin llegar a resolver la operatoria.

Ambos tipos de investigación (Baron-Cohen et al., 1986, y Rivière y Castellanos, en prensa) coinciden en una caracterización especial del autismo, y al mismo tiempo proporcionan una información muy interesante sobre el funcionamiento del niño normal. Estos resultados sugieren la hipótesis según la cual la diferenciación de dominios caracterizaría el desarrollo normal, y su alteración podría estar relacionada con trastornos graves de la relación podría estar relacionada con trastornos graves de la relación y la comunicación, como los 
propios del autismo. Idea que ya ha sido sugerida anteriormente desde un paradigma y área de investigación muy diferente (Minsky, 1986).

«Cómo inician los niños su camino hacia la diferenciación de relaciones psicológicas y físicas? En el apéndice habré de sugerir que nuestro cerebro infantil está genéticamente equipado con mecanismos que facilitan el aprendizaje de las señales sociales. Pero, ¿qué pasaría si esos mecanismos fallaran de alguna manera, de modo que por casualidad (o por descuido o por accidente) no llegara a producirse esa división en ámbitos? Entonces todas esas diferentes clases de pensamiento se fundirían en una sola, y el niño se enfrentaría a la imposible tarea de formular principios aplicables a todos los terrenos. Si un niño intentara ver el mundo sin dividirlo en ámbitos, no lograría encontrar reglas simples que fueran aplicables a una gama tan variada de realidades.

"... Esta podría ser la razón de algunos aspectos de los desórdenes de los niños que los psiquiatras llaman "autistas". Estos desafortunados individuos no establecen comunicación afectiva con otras personas, si bien son capaces de adquirir cierta competencia en el manejo de las cosas físicas. Nadie conoce la causa de tales desórdenes.»

(Minsky, 1986, p. 309 de la trad. cast.)

\section{CONCLUSIONES}

En conclusión, la escasez de correlaciones entre las escalas cognitivas y las funciones y conductas comunicativas observadas, su dispersión entre las diversas escalas y la incosistencia de las correlaciones al comparar los resultados de las dos situaciones de observación planteadas, así como al compararlos con los de la investigación de Bates et al. (1979), no confirman la hipótesis de homología que defiende la interdependencia estructural entre las capacidades comunicativas y las capacidades sensoriomotrices de adaptación al mundo físico, ni confirman tampoco el patrón de relaciones específicas propuesto por Bates et al. (1979) a partir de los resultados de su investigación, que le llevaron a plantear la hipótesis de homología local.

Por otra parte, los resultados de los análisis de correspondencias múltiples, y concretamente la notable presencia de factores compuestos exclusivamente por variables de un mismo dominio (cognitivo o comunicativo) no apoyan la hipótesis de la existencia de estructuras específicas compartidas entre determinadas capacidades vinculadas al dominio del mundo físico y las capacidades de adaptación al mundo social, más bien sugieren una hipótesis de diferenciación de los dominios social-comunicativo y físico en el período de aparición de la intención comunicativa.

Aunque los resultados de esta investigación sólo pueden sugerir y no permiten aún superar la reflexión crítica de Schaffer (1984) sobre la insuficiencia de datos empíricos para afirmar la existencia de dos mundos diferenciados en el desarrollo del niño (el social y el físico), vienen a sumarse a un conjunto de investigaciones, cada vez más amplio, que de una forma u otra detectan estas diferencias y valoran su importancia para la comprensión del desarrollo en este período tan relevante. 


\section{LISTA DE SIMBOLOS}

1. «Ordinal Scales of Psychological Development», de Uzgiris y Hunt (1975)

I: Escala de permanencia del objeto

II: Escala de relaciones medios-fines

IIIA: Escala de imitación verbal

IIIB: Escala de imitación gestual
IV: Escala de causalidad operativa

V: Escala de relaciones espaciales

VI: Escala de esquemas sensoriomotores

2. Categorías de observación:

ACI: Acto comunicativo intencional

$\mathrm{R}$ : Respuestas

2.1. Funciones comunicativas:

DO: Demanda de objeto

DA: Demanda de ayuda

JIO: Juego interactivo con objeto

JISO: Juego interactivo sin objeto

NO: Rechazos y protestas

PD: Protodeclarativos

2.2. Conductas comunicativas:

CO: Contacto ocular

MA: Mirada alterna

ES: Estira el brazo hacia

SE: Señala con el índice

TA: Toca al adulto

TO: Toca el objeto

EN: Entrega

OF: Ofrece

APL: Aplica el objeto
MU: Muestra
AP: Aparta
SI: Simula
GC: Gesto convencional
VO: Vocalizaciones
EV: Emisión verbal
PRO: Agitación «procedimiento»

\section{Referencias}

Ainsworth, M. D. S. y BELL, S. (1974): Mother-infant interaction and the development of competence. En K. J. Connolly y J. Bruner (Comps.): The growth of competence. Nueva York Academic Press.

BaKeman, R. y GotTMAN, J. M. (1986): Observing interaction: an introduction to sequential analysis. Cambridge, Cambridge University Press. (Trad. cast.: Observación de la interacción social: introducción al análisis secuencial Madrid, Morata. 1989).

Baron.Cohen, S.; LesLie, A. M. y Frith, U. (1986): Mechanical, behavioral and intentional understanding of pictures stories in autistic children. British Joumal of Developmental Psychology, 4, 113-125.

Bates, E.; Camaioni, L. y Volterra, V. (1975): The acquisition of performatives prior to speech. Merrill-Palmer Quarterly, 21, 205-226.

Bates, E.; Camaioni, L. y Volterra, V. (1976): Sensoriomotor performatives. En E. Bates: Language and context: the acquisition of pragmatics. Nueva York, Academic Press, 49-71.

Bates, E.; Benigni, L.; Bretherton, I.; Camaioni, L. y Volterra, V. (1977): From gesture to the first word: on cognitive and social prerequisites. En M. Lewis y L. A. Rosenblaum (Comps.) Interaction conversation and the development of language. Nueva York, Wiley, 247-308.

Bates, E.; Benigni, L.; Bretherton, I.; Camaioni, L. y Volterra, V. (1979): Cognition and Communication from nine to thirteen months: Correlational findings. En, E. Bates, L. Benigni, L. Camaioni, I. Bretherton y V. Volterra (Comps). The Emergence of Symbols. Cognition and communication in infancy. Nueva York, Academic Press, 69-140.

Bates, E. y Synder, L. (1987): The cognitive hypothesis in language development. En I. C. Uzgiris y J. M. Hunt (Comps.): Infant Performance and Experience: New findings with the Ordinal Scales: Urbana, University of Illinois Press.

BELL, S. M. (1970): The development of the concept of the object as related to infant mother attachment. Cbild Development, 41, 291-313. 
Blanco, A. y Anguera, M. T. (1984): Fiabilidad, precisión y validez de los requisitos observacionales. Comunicación presentada en el XXIII Congreso Internacional de Psicología. Acapulco, Méjico.

Camaioni, L., Volterra, V. y Bates, E. (1976): La comunicazione nel primo anno di vita. Torino, Boringhieri.

Carranza, J. A., Pérez, J. y Brito de la Nuez, A. (1985): Noción de intermediario y actos comuncativos intencionales en niños prelingüísticos. Anuario de Psicología, 32, 58-72.

CARTER, A. L. (1974): The development of communication in the sensorimotor period: a case study. Doctoral dissertation, no publicada. University of California-Berkeley.

Curcio, F. (1978): Sensorimotor functioning and communication in mute autistic children. Journal of Autism and Childhood Schizophrenia, 8, 281-292.

Dore, J. (1973): The development of speech acts. Doctoral dissertation, no publicada. City University of New York.

GÓMEZ, J. C. (1990): The emergence of intentional communication as a problem-solving strategy in the gorilla. En S. T. Parker y K. R. Gibson (Comps.): Language and Intelligence in Animals: Developmental Perspectives. Cambridge University Press.

GONZÁlEZ DEL YeRRO, A. (1986): La percepción de contingencias como determinante del conocimiento social temprano: reacciones de neonatos ante contingencias sociales y no sociales. Memoria de Licenciatura no publicada. Universidad Autónoma de Madrid.

Harding, C. G. (1982): Development of intention to communicate. Human Development, $25,140-151$.

HARDing, C. G. y GounkofF, R. M. (1979): The origins of intentional vocalizations in prelinguistic infants. Child development, 50, 33-40.

Hogan, A. E. (1983): Use of confirmatory factor analysis to examine relationships among sensoriomotor communication and social skills in infancy. Doctoral dissertation, no publicada. University of Miami.

JaCkON, E.; CAMpos, J. y Fischer, K. (1978): The question of decalage between object permanence and person permanence. Developmental Psychology, 14, 1-10.

Lebart, L. y Morineau, A. (1984): SPAD, Tomo 3. París, CESIA.

Martisen, H. y Von Tetzchner, S. (1988): Development of intentional communication. Comunicación presentada en Third European Conference of Developmental Psychology. Budapest.

MinsKy, M. (1986): The Society of Mind. Nueva York, Simon y Schuster. (Trad. cast.: La sociedad de la mente. Buenos Aires, Ediciones Galápago).

Mundy, P. C. y SEIBERT, J. M. (1980): Procedures, scoring sheets and profile for the Adapted Uzgiris-Hunt Scales. Manuscrito no publicado. Mailman Center for Child Development, University of Miami, Florida.

Paradise, E. B. y Curcio, F. (1974): Relationships of cognitive and affective behaviors to fear strangers in male infants. developmental Psychology, 10, 476-483.

Piaget, J. (1936): La naissance de l'intelligence chez l'enfant. París, Delachaux et Niestlé. (Trad. cast.: El nacimiento de la inteligencia en el niño. Madrid, Aguilar, 1969).

Piaget, J. (1937): La construction de réal chez l'enfant. París, Delachaux et Niestlé. (Trad. cast.: La construcción de lo real en el niño. Barcelona, Ed. Crítica, 1985).

Rivière, A. y Castellanos, J. L. (en prensa): Conocimiento impersonal y comprensión interpersonal en autismo.

SAINT:PIERRE, J. (1962): Evide des differences entre la recherche active de la personne bumaine et celle de l'objet inanimé. Tesis de Master, Universidad de Montreal.

$\mathrm{S}_{\mathrm{ARRIA}}$, E. (1989): La intención comunicativa preverbal: observación y aspectos explicativos. Tesis Doctoral no publicada. Madrid, UNED.

SARRIÁ, E. (1991): Observación de la comunicación intencional preverbal: un sistema de codificación basado en el concepto de categoría natural. Psicothema, vol. $3, \mathrm{n} .^{\circ} 2$.

ScOTT, W. A. (1955): Reliability of content analysis: the case of nominal scales coding. $P u$ blic Opinion Quarterly, 19 (3), 321-325.

Schaffer, H. R. (1984): The child's entry into a social world. Londres, Academic Press.

SEIBERT, J. M. (1976): The infant's differential understanding of person permanence and object permanence: decalage or artifact? Doctoral thesis, no publicada. University of Illinois at Urbana-Champaign.

SEIBERT, J. M. y Hogan, A. E. (1982): Procedures Manual for the Early Social Communication Scales (ESCS). Manuscrito no publicado. Mailman Center for Child Development, University of Miami.

Sugarman, S. A. (1973): A description of communicative development in the prelanguage children. Honors Thesis, Hampshire College.

Sugarman-Bell, S. A. (1978): Some organizational aspects of preverbal communications. En I. Markova (Comp.): The social context of language. Nueva York Wiley, 49-67.

Sugarman, S. A. (1984): The Development of Preverbal Communication: its contribution and limits in promoting the development of language. En R. L. Schiefelbusch y J. Pickar (Comps.): The Acquisition of Communicative Competence. Baltimora, University Park Press, 23-68.

UzGiris, I. y Hunt, J. McV. (1975): Assesment in infancy. Urbana, University of Illinois Press. 